\title{
Un gène de l'achondroplasie/hypochondroplasie est localisé sur l'extrémité du bras court du chromosome 4
}

L'achondroplasie, connue depuis l'Antiquité (dieu Ptah) et identifiée par Parrot en 1878[1] est un nanisme caractérisé par une micromélie* et une macrocéphalie**, dues à une anomalie du cartilage de croissance. La zone sériée du cartilage conjugal est pauvre et fibreuse, et la cause de cette anomalie du développement osseux est encore très mystérieuse $[2,3]$. Cette affection est fréquente, touchant un enfant sur 15000 ou 20000 , reconnue dès la naissance par une disproportion entre les membres et le tronc, contrastant avec une insuffisance de taille modérée et, à ce stade, des lésions radiologiques caractéristiques : extrémités fémorales supérieures claires, ailes iliaques courtes, élargissement métaphysaire. L'évolution, dans les premières années, est caractérisée par une augmentation du périmètre crânien (liée à une dilatation modérée des ventricules cérébraux, conséquence d'un rétrécissement du trou déchiré postérieur) qui se stabilise au cours de la seconde année. Le retentissement sur la taille dépend du défaut de croissance des membres alors que le tronc évolue normalement. Dans l'enfance, les signes radiologiques discriminants sont la micromélie, les os longs trapus au relief saillant, les ailes iliaques courtes et carrées, un col fémoral court ; la réduction des distances interpédiculaires, témoins de pédicules courts et les vertèbres réduites dans leur dimension antéropostérieure sont très caractéristiques. Cette affection est autosomique dominante; la pénétrance est considérée comme complète et elle apparaît le plus souvent isolément

\footnotetext{
* Micromélie: malformation caractérisée par la diminution de taille d'un ou de plusieurs membres. ** Macocéphalie: augmentation du volume de la
}

dans des familles saines par mutation récente (8 cas sur 10). Un âge paternel moyen élevé a été montré par plusieurs auteurs dans les cas sporadiques, témoignant, avec d'autres affections, de l'effet de l'âge du père sur l'apparition de mutations dominantes [4].

La sévérité de l'insuffisance de taille semble jouer un rôle dans la dimension des familles avec plusieurs personnes atteintes. Nous avons pu collecter six familles multiplex, de dimension modeste, insuffisantes pour mener à bien une étude de liaison avec des microsatellites anonymes du Généthon [5], régulièrement répartis sur le génome.

Il nous a paru alors judicieux d'adjoindre à cet échantillon des familles d'hypochondroplasie [6]. Cette affection, identifiée plus récemment, est cliniquement et histologiquement très voisine de l'achondroplasie, réalisant dans notre expérience clinique un réel continuum. Ainsi, quinze familles ont pu être collectées, comportant 44 individus atteints et 35 apparentés. Des études ponctuelles avaient déjà été réalisées, faisant rejeter une liaison avec certains constituants du collagène $[7,8]$ et des protéoglycanes. La construction d'une carte génétique d'exclusion a permis d'éliminer la responsabilité des chromosomes 1 et $12 q$ ainsi qu'une liaison avec 21 autres marqueurs répartis sur les autres autosomes. Très récemment, cependant, nous avons pu montrer, avec une équipe américaine qui avait entrepris une étude similaire, une liaison avec D4S412, un marqueur du chromosome 4 [4]. L'utilisation d'autres marqueurs de la région toute télomérique du chromosome $4 \mathrm{p}$ a mis en évidence une liaison avec le locus de l'iduronidase A (lod-score maximal $=4,71$ à $\theta=0$ ) [9]. L'analyse multipoint des données confirme la localisation très distale sur le chromosome 4 [10]. Il est important de souligner qu'un lod-score à 3,01 à $\theta=0$ est calculé sur le seul échantillon de familles atteintes d'achondroplasie et que les tests statistiques montrent une homogénéité génétique de cette maladie et de l'hypochondroplasie. Ce premier pas permet d'espérer l'identification, dans un proche avenir, du gène responsable de ces affections et la mise en évidence des mécanismes à l'origine du plus fréquent des nanismes.

M.L.

F.R.

A.M.

P.M.

1. Parrot JM. Sur les malf ormations achondroplasiques et le dieu Ptah. Paris, Bull Anthropol 1878; 1: 296.

2. Maroteaux P, I amy M. Achondroplasia in man and animals. Clin (rthop 1964; 33: 91-103.

3. Stanescu V, Stanescu R, Maroteaux P. Etude morphologique et biochimique du cartilage de croissance dans les osteochondrodysplasies. Arch lir Pediatr 1977; 34: (suppl 3). 4. Weissenbach J, Gyapay (;, Dib (,, Vignal A, Morissette J, Milasseau P, Vaysseix G, Lathrop $M$. A second generation linkage map of the human genome. Nature 1992; 359: 794-801

5. Gardner RJM. A new estimate of the achondroplasia mutation rate. Clin (ienet 1977; 11: 31-8.

6. Maroteaux P, Falzon P. Hypochondroplasie. Revue de 80 cas. Arch Fr Pediatr 1988; 45 : 105-9.

7. Ogilvie D, Wordsworth P, Thompson E, Sykes B. Evidence against the structural gene encoding type II collagen (COL.2AI) as the mutant loms in achondroplasia. J Med Genet $1986 ; 23: 19-22$.

8. Carter WA, et al. SSC.P and segregation analysis of the human type $X$ collagen gene (C.OL.10Al) in heritable forms of chondrodysplasia. Am / Hum Genet 1992 ; 51 : 841-9.

9. Lathrop GM, Lalouel JM. Easy calculation of lod scores and genetic risk on small computers. Am J Hum Genet 1984; 36: 460)-5. 10. Le Merrer M, Rousseau F, Legeai-Mallet L. A gene for achondroplasia-hypochondroplasia maps to chromosome 4p. Nature (ienet 1994; 6 : 318-21. 